\title{
Matrix Cracking in Non-symmetric Laminates under Combined Membrane and Flexural Loading
}

\author{
A. Adumitroaie, E. J. Barbero, and M. Schagerl
}

\begin{abstract}
A constitutive model of progressive matrix cracking in fiber reinforced laminates is developed for the case of both membrane and flexural deformation. The analytical progressive damage model addresses both the degraded mechanical properties of the laminate for given levels of matrix cracking in individual plies, and the damage growth under applied loading.

Crack densities in individual plies are the damage state variables of the model. This formulation is unlike the progressive damage models for composites implemented in most of the FEA packages, where softening laws are considered in order to describe stiffness reduction and damage evolution. By using the ply crack densities as state variables the model is able to predict and to keep track of the crack density in individual plies during the loading history, which can be of interest in application where the permeability (leakage) of the laminate is a limiting design factor. One example can be pressure vessels containing fluids or gases. Thermal residual stresses are taken into account in the present model, which extends the predictive capabilities of the model to applications in the range of cryogenic temperatures.

The analytical model is validated against available experimental data for the case of both membrane and flexural loading.
\end{abstract}

Index Terms-Composite material, matrix cracking, progressive damage.

\section{INTRODUCTION}

The characterization of composites has been an intensive field of research since their emergence as a new class of high performance structural materials. On one hand, the advantages offered by composite materials are very tempting, opening a large pool of choices for both new applications and alternative solutions for traditional applications, e.g., lightweight structures. On the other hand, the use of composite materials requires knowledge of limiting conditions under lifetime loading for each application, and this knowledge requires extensive amount of research. As a matter of fact, the same anisotropy and heterogeneity of the material properties that governs their unique behavior, also raises the complexity in quantifying and understanding the behavior and the properties. Thus, new experimental and analytical tools are constantly evolving to study composite materials.

Manuscript received March 9, 2015; revised October 9, 2015.

A. Adumitroaie and M. Schagerl are with the Institute for Constructional Lightweight Design, Christian Doppler Laboratory for Structural Strength Control of Lightweight Constructions, Johannes Kepler University, 4040 Linz, Austria (e-mail: adi.adumitroaie@jku.at, martin.schagerl@jku.at).

E. J. Barbero is with Mechanical and Aerospace Engineering, West Virginia University, Morgantown, WV 26506-6106, USA (e-mail: ever.barbero@wvu.edu).
Damage mechanisms such as fiber-matrix debonding, fiber breaking, fiber micro-buckling, matrix cracking, inter-ply delamination, follow different laws particular of the material structure and loading case. These modes can be catastrophic (i.e., structural failure) or not, but regardless of their primary structural impact, each one has to be very well understood and controlled. All of them can cause deterioration in the mechanical property and structural integrity, and in some cases they interact, as it is the case when a non-catastrophic damage mode may work as a nucleation point of a catastrophic damage mode.

It is also the case that failure is defined not only considering the ultimate structural load carrying capacity, but also considering the functionality of the structure. In this way, some damage mode that is usually considered as non-catastrophic, e.g. matrix cracking can become a limiting one. This case can be best exemplified for the case of a pressure vessel: failure might be defined when the first crack appears and the structure stops meeting its functional requirements, i.e., containing the pressurized fluid or gas. Thus, matrix cracking can be regarded as a limiting mode of damage in some applications where it can constitute a path way for gas or liquid penetration, leading to exterior leakage or even the more difficult to detect fibers corrosion. The latter may induce weakening of fibers and ultimately fiber breakage.

The matrix cracking damage mode in laminated fiber reinforced polymers (FRP) has been studied extensively by the composite research community. The first analytical models were developed for the classical example of the $\left[0_{m} / 90_{n}\right]_{S}$ cross-ply laminate [1]-[3], featuring cracks in the transverse central $90^{\circ}$ plies. Then, the study was extended to more general cases, like: $\left[S / 90_{n}\right]_{S}$ laminates [3]-[6], where the letter $S$ denotes a sub-laminate (not necessarily $0^{\circ}$ orientation) that does not undergo matrix cracking under the considered loading case; $[S / 90 / S / 90 / \ldots / S / 90]_{S}$ laminates, featuring matrix cracking in multiple transverse $90^{\circ}$ plies; $\left[S / \theta_{1} / \theta_{2}\right]_{S}$ laminates [7]-[9], where the loading case is such that matrix cracking develops in the two off-axis $\theta$ plies; $\left[ \pm \theta / 90_{n}\right] s$ laminates [10], [11], where matrix cracking can develop in both $\theta$ and $90^{\circ}$ plies; $\left[\theta_{1} / \theta_{2} / \ldots / \theta_{n}\right]_{S}$ laminates [12], [13], where matrix cracking can appear in all off-axis $\theta$ plies. A common limiting characteristic of these models is the fact that the laminate has to be symmetric, and to preserve the symmetry after damage onset. This constrain on the laminate configuration brings, in turn, a constrain on the loading case: flexural loading cannot be addressed, since in that case matrix cracking appears only in those plies loaded in tension, and the symmetry of the laminate is lost. Thus, most of the existing models in the literature deal only with symmetric 
laminates under in-plane loading. Very often, even this case is further constrained to uni-axial loading, in order to avoid generating damage in the aforementioned $S$ sub-laminates.

Models considering the flexural deformation are even rarer in the literature, and, in most of the cases, considering bending deformation brings additional restrictions on the level of generality and applicability of the solution. Again, only particular LSS can be addressed; or, due to some build--in modeling assumptions, all damaged plies must have the same matrix crack density, condition which cannot be satisfied if there are multiple cracking plies under flexural loading.

Numerical solutions, especially the FEA method, can be an approach alternative to analytical models, for solving the complex $3 D$ stress-strain fields, of the matrix cracking in laminated composites [14]-[16]. Theoretically it is possible to solve the problem of the reduced material properties by FEA method, but these detailed analyzes apply to particular laminate configurations. A parametric study using FEA is difficult to implement, involving multiple meshing. We also have to remember that a real problem, of a real structure considering loading, material properties and boundary conditions as required by the FEA method, becomes a double FEM problem. First of all, the whole structure is discretized and meshed to find the FEA solution for the considered loading case and boundary conditions. Then, when the resulting nonuniform stress field locally reaches the level of damage growth, a second FEA problem has to be solved, to provide the new (reduced) material properties at the laminate scale, for the corresponding extent of damage at the local (ply and material point) level. The double FEM problem continues for each of the external loading increments. The double meshing process becomes very cumbersome and expensive computationally. An easier way to solve the problem [17] consists of having an analytical solution for the problem of reduced material properties at a certain damage level (reduced material model), and for the problem of damage extent at a certain loading level (damage growth model). The analytical solution is then provided to the FEA problem of the real structure under loading and boundary conditions: the material properties of the individual finite element integration points are modified according to the analytical solution of the reduced material model and damage growth model.

The objective of the work presented here is the development of an analytical model for matrix cracking progressive damage in composites of general laminate stacking sequence (LSS) under generalized membrane/flexural deformation. Both problems, the reduced material model and the damage growth model are addressed. There is no limitation on the configuration of the laminate or on the number of the cracking plies. Various other aspects that can influence the matrix cracking process are considered and included in the present model: the effect of thermal residual stress; the possible fracture modes $I, I I$, or mixed I-II; the possibility of crack closure under flexural deformation; the experimentally observed strengthening of the material (i.e., increase resistance of the material to damage growth) with increasing damage level, usually referred to as Resistance-curve (R-curve) behavior; the in-situ constraining influence of the neighboring plies on the progressive damage of the cracking ply, and the influence of the thickness of the cracking ply on damage initiation and progression.

The proposed progressive damage model makes use of the following key ingredients: i) an appropriate $C O D$ - based reduced material model [14], [18]; ii) an energy based damage evolution criterion inspired by Fracture Mechanics; iii) an homogenization technique inspired by $C D M$; iv) an iterative procedure in order to detect the conditions for damage growth in individual plies of the laminate, and to increase the damage level when the conditions are met; and v) the Classical Laminate Theory (CLT) [19] in order to describe the overall membrane and flexural deformation of the laminated composite.

The model aims to releasing the limiting assumptions used in previous models dealing with the same problem. The resulting analytical model is amenable to easy implementation in a FE formulation, as a nonlinear material behavior, which is able to predict the onset and evolution of matrix cracking damage in laminated composites, and to assess the effect of this damage mode on the material and structural response.

\section{ANALytiCAl MODEl Formulation}

The area of applicability of the model naturally comes from the main hypotheses under which the model is developed:

- aligned long fiber reinforced laminated composites; matrix cracking takes place in individual plies along the fiber direction;

- linear elastic material behavior up to damage onset; the possible material nonlinearity due to matrix plastic deformation is not considered;

- brittle fracture behavior; there is no energy released for the formation of plastic zones; the progressive damage growth is modeled under the frame of linear elastic fracture mechanics (LEFM);

- propagated cracks; one matrix crack suddenly develops and spans the whole width of the specimen; the progressive damage growth is rather a discrete process characterized by discrete fracture events, than a continuous one;

- there is uniform spatial distribution of the propagated cracks in a ply affected by matrix cracking; cracks are equally spaced between them;

- there is a strengthening effect (R-curve behavior) of the damage growth on the critical parameter, i.e., the critical strain energy release rate (ERR) Gc, characterizing the material resistance to crack growth;

- the R-curve behavior features an in-situ nature (i.e., ply thickness dependency).

The main ingredients and modules of the model, and the basic principles of how they work and interact together are described in the following:

Reduced Material Properties — are approached through the $C O D$-based material model in [14]. Here, the overall laminate thermo-elastic properties for a given damage level (crack density) in each ply of the laminate can be calculated; however, no information can be recovered with regard to 
individual plies properties (ply reduced stiffness and ply reduced thermal expansion coefficients), which means that the reduced material model in [14] features a laminate formulation. For the present model, individual reduced plies properties are needed in order to calculate the strain energy required by the energy based damage evolution function. This fact is achieved by the iterative procedure of the model.

A ply homogenization technique — inspired by the CDM. In this procedure, individual plies having a certain damage level (crack density) are regarded as fictitious homogenized materials having as effective thermo-elastic properties the properties of the cracked ply. Once the reduced ply properties are determined, individual ply cracks are not physically considered, but the ply cracks are rather regarded as being smeared through the volume of the ply.

An iterative procedure - meant to detect the damaging ply $(k 0)$ ply in the present notation) at a certain level of loading, according to the damage evolution criterion, and to allow the incremental increase in damage in this $(k 0)$ ply. Furthermore, after the $(k 0)$ ply is given one damage increment, the iterative procedure (also regarded as LSS iteration in the following) repeatedly iterates over the stacking sequence of the laminate at the same load level, in order to check for possible multiple increments in damage level of the same damaging ply $(k 0)$, or to check for multiple cracking plies at the same load level, due to stress redistribution after the damage level in the initial detected $(k 0)$ ply is increased and the ply stiffness is accordingly reduced.

Damage Evolution - is approached through a fracture mechanics inspired Energy Release Rate criterion. The damaging ply $(k 0)$ is detected by the damage evolution criterion together with the iterative procedure, and the damage level is incrementally increased in this ply.

Classical Laminate Theory (CLT) - provides the overall, effective constitutive behavior of the laminate containing cracks in individual plies, provided that the homogenized, effective ply properties are known.

As stated before, two main aspects have to be considered in order to perform progressive damage analysis of laminated composites. First, the reduction in the material thermo-elastic constants at a given damage level has to be evaluated; this aspect is regarded as the reduced material properties in the present model, and can be described by the relationship

$$
[C]=\left\lfloor C\left(\lambda^{(k)}\right)\right\rfloor
$$

where $[C]$ generically represents the thermo-elastic properties of the material, and $\lambda$ represents the state variable accounting for the damage level inside of the material. For the present model of intra-laminar matrix cracking, the damage variable is crack density (i.e., number of cracks per unit length $\lambda^{(k)}=1 / d^{(k)}$, where $d^{(k)}$ is the distance between two consecutive cracks inside of any ply $(k)$ of the laminate. The second aspect is the prediction of the damage onset and progression due external thermo-mechanical loads applied to the laminate. This aspect is regarded as damage growth, and it can be represented by the relationship

$$
\lambda^{(k)}=\lambda^{(k)}(\varepsilon)
$$

where $\varepsilon$ generically represents the thermo-mechanical deformation of the laminate.

\section{LAMinAte REDUCED MATERIAL PROPERTIES}

The reduced material model in [14] is implemented in the present progressive damage model. For the uncracked material, a stress state $\sigma_{i j}^{(k)}, i, j=1 \cdots 3$ exists in each ply $(k)$ of the laminate, for a given deformation state of the laminate. The stress state $\sigma_{i j}^{(k)}$ results in a corresponding vector $\tau_{i}^{(k)}, i=1 \cdots 3$ of surface tractions at the prospective location of the crack in each ply of the laminate, according to the Cauchy stress resultant formula of classical elasticity:

$$
\tau_{i}=\sigma_{\mathrm{ij}} \cdot n_{j}
$$

where $n_{j}$ is the vector of the direction cosines of the normal to the crack surface. Thus, the correspondence between the surface tractions $\tau_{i}$ and the fracture mechanics damage modes is: $i=1$-crack opening, $i=2$-crack shearing or $i=$ 3 -crack tearing. Due to the fact that cracks always appear along the fiber direction (i.e., the 1 direction in the c.s. of the ply), the normal direction $n$ is always along the 2 transverse direction of the cracking ply.

The average crack displacement of cracks in ply $(k), \Delta u_{i}^{(k)}$, represents the relative displacement between individual faces of the crack, corresponding to $i=1,2,3$ modes. The problem of $\Delta u_{i}^{(k)}$ is regarded as the superposition of two separate elasticity problems: first the surface tractions $\tau_{i}$ on the prospective crack surface are calculated for the material regarded as uncracked; then the average crack displacement in ply $(k)$ is considered as the result of the linear contribution of surface tractions in all plies of the laminate $\tau^{(l)}(l=1 \cdots N)$, where $N$ is the number of plies in laminate. In this way, the average crack displacement in ply (k) becomes

$$
\Delta u_{i}^{(k)}=t^{(k)} \sum_{l=1}^{N} \beta_{i j}^{(k l)} \cdot \tau_{j}^{(l)}, \quad i, j=1 \cdots 3
$$

where $t^{(k)}$ is the ply thickness. It can be observed that the linear combination in (4) is made through the coefficients $\beta^{(k l)}$, which quantify the effect of surface traction in ply $(l)$ on the COD in ply $(k)$. The coefficients $\beta_{i j}^{(k l)}$, also called COD coefficients, are function of: (i) LSS configuration, (ii) ply material properties, and (iii) ply crack density (if cracks influence or not each other); they become now the new unknowns of the problem. The physical meaning of the $\beta_{i j}^{(k l)}$ coefficients is not a direct intuitive one; they act as 
compliance between the surface tractions in ply $(l), \tau_{j}^{(l)}$, and the resulting COD in ply $(k), \Delta u_{i}^{(k)}$, in the linear combination in (4); they are similar to influence coefficients in [20, Appendix].

There is no direct analytical solution for the COD parameters $\beta_{i j}^{(k l)}$ in (4) for laminated composites. At this point, the approximation of using $C O D$ analytical solutions to a closely related, yet different problem, i.e., the fracture mechanics problem of a row of cracks in an infinite/semi-infinite orthotropic plate, is proposed in [21]-[23]. The level of error introduced by this approximation is checked by comparing the output of the analytical model with experimental data and extensive FE simulations on a large variety of laminate types, and a good correlation is found. Moreover, for the cases where the fracture mechanics solution is not available, analytical laws of COD are obtained by extrapolating extensive parametric FE analyzes [22].

Next, an energy balance equation between two equivalent instances of the cracked laminate is applied [14], [18]:

First, the elastic energy $W_{c}$ stored in the composite material featuring matrix cracks is calculated as a linear elasticity superposition problem:

$$
W_{c}=W_{0}+\Delta W
$$

where $W_{0}$ is the elastic energy in the uncracked material, and $\Delta W$ is the change in the elastic energy due to advent of matrix cracks. The change in elastic energy $\Delta W$ is calculated based on the LEFM Irwin principle (which states that the energy released equals the mechanical work for crack closure) as the mechanical work done by the surface tractions

$\tau_{i}$ (3) at the prospective crack location, through the corresponding COD $\Delta u_{i}(4)$.

Second, the cracked laminate is considered in its effective (homogenized) instance, and the same $W_{c}$ in (5) is expressed in terms of effective (reduced) material properties $C_{c}, \alpha_{c}$ :

$$
W_{c}=W_{c}\left(C_{c}, \alpha_{c}\right)
$$

where $C_{c}$ and $\alpha_{c}$ are the reduced stiffness and the reduced CTE of the cracked laminate, respectively.

By identification of the two expressions (5) and (6) of the stored energy $W_{c}$ in the damaged material, the reduced thermo-elastic material properties $C_{c}, \alpha_{c}$ can be calculated as closed form analytical solutions:

$$
\left[C_{c}\right]_{6 \times 6}=\left[C_{c}\left(\rho^{(k)}\right)\right], \quad\left\{\alpha_{c}\right\}_{6 \times 1}=\left\{\alpha_{c}\left(\rho^{(k)}\right)\right\}
$$

where $\rho^{(k)}$ is the normalized crack density in each ply $(k)(k$ $=1 \ldots N)$ of the laminate. The normalized crack density is defined as $\rho^{(k)}=t^{(k)} / d^{(k)}=t^{(k)} \cdot \lambda^{(k)}$; the normalized crack density is a measure of ply damage level equivalent to the crack density $\lambda^{(k)}$ in eq. (1). The $6 \times 6$ and $6 \times 1$ subscripts in eq. (7) indicate that the reduced effective (laminate) material properties are for the case of combined membrane and flexural deformation. In classical notation of $C L T$, the reduced stiffness $C_{c}$ in eq. (7) is the $[A B D]$ membrane-flexural stiffness matrix.

This method, based on the concept of COD, provides a convenient closed form solution to the problem of reduction in material properties of the general $\left[\theta_{1} / \theta_{2} / \ldots / \theta_{n}\right]_{S}$ laminate affected by matrix cracks in any of its plies. The main achievements of this approach is that the restriction on the number of cracking plies is released, and the whole set of thermo-elastic constants in eq. (7) can be evaluated, using as only input the uncracked plies properties and the crack density $\rho^{(k)}$ in each ply of the laminate. A detailed derivation and explanation of the laminate reduced material model can be found in [14], [24].

\section{Ply Reduced Material Properties}

The thermo-elastic properties of individual plies affected by matrix cracking are calculated inside of the iterative procedure (or LSS iteration). First, at any given load increment, the iterative procedure checks for the cracking ply (noted $(k 0)$ ) inside of the laminate; the cracking ply is identified as being the ply which first meets the condition of the damage evolution criterion in eq. (11). When a $(k 0)$ cracking ply is found, the crack density level in that ply is incrementally increased at a subsequent value

$$
\rho_{i+1}^{(k 0)}=\rho_{i}^{(k 0)}+\Delta \rho_{i}
$$

Then, the new effective laminate properties are calculated by the reduced material model (7). At this point, the iterative procedure provides a means of calculating the reduction in individual properties of the cracking ply $(k 0)$ : considering that the reduction in effective laminate properties are only due to increasing the crack density in the $(k 0)$ ply, the new effective material properties of the ply $(k 0)$ are calculated through the $C L T$ as

$$
\begin{aligned}
& {\left[Q^{(k 0)}\right]=\frac{1}{t^{(k 0)}}\left\lfloor\left[A^{(k 0)}\right]-\sum_{k=1}^{N}\left(1-\delta_{k, k 0}\right) \cdot t^{(k)} \cdot\left[Q^{(k)}\right]\right]} \\
& \left\{\alpha^{(k 0)}\right\}=\frac{1}{t^{(k 0)}}\left[Q^{(k 0)}\right]^{-1} \cdot \\
& \cdot\left[-\left\{\delta_{E}^{(k 0)}\right\}-\sum_{k=1}^{N}\left(1-\delta_{k, k 0}\right) \cdot t^{(k)} \cdot\left[Q^{(k)}\right] \cdot\left\{\alpha^{(k)}\right\}\right]
\end{aligned}
$$

where $\left[A^{(k 0)}\right]$ and $\left\{\delta_{E}^{(k 0)}\right\}$ are respectively the effective (reduced) laminate in-plane stiffness matrix and the effective laminate in-plane vector of thermal effect coefficients, provided by the material model (7); $\delta_{k, k 0}$ is the Kroneker-Delta function, which returns the value 1 if $k=k 0$, 
or value 0 if $k \neq k 0$. It has to be noted that the values $\left[A^{(k 0)}\right]$, $\left\{\delta_{E}^{(k 0)}\right\}$ are overall laminate properties; the subscript $(k 0)$ has been used for them only to indicate that they are calculated due to a crack multiplication in $(k 0)$ ply only.

After both effective laminate properties (7), and the effective ply properties (9) are calculated, the iterative procedure is resumed in order to check for other cracking plies at the same level of loading, or for multiple crack increments (8) for the same cracking ply $(k 0)$. The iteration stops when no ply meets the condition in the damage evolution criterion.

Regarding the increment in crack density $\Delta \rho$ in eq. (8), different modeling approaches can be considered: an infinitesimal increase in crack density at each cracking event; a discrete increase in crack density corresponding to the formation of one more crack; or a discrete doubling in crack density. The progressive damage model proposed here uses the approach of 1 more crack discrete crack multiplication process, which provides the incremental value of crack density in eq. (8) [24], [25].

$$
\rho_{i+1}=(i+2) \rho_{0}
$$

where $\rho_{0}$ is the initial starting value of the crack density, corresponding to an arbitrary initially very large crack spacing between preexisting cracks in all plies of the laminate, in the undamaged state.

\section{Progressive Damage}

A damage evolution criterion is required in order to detect the conditions for matrix crack multiplication in individual plies of the laminate under loading. In the present formulation, a fracture mechanics inspired criterion [26] is selected, in order to consider mixed modes crack formation for the case of matrix cracking under general in-plane and flexural deformation.

$$
g_{(k 0)}=\left(1-\frac{G_{I c}}{G_{I I c}}\right) \sqrt{\frac{G_{I}^{(k 0)}}{G_{I c}}}+\frac{G_{I}^{(k 0)}}{G_{I I c}}+\frac{G_{I I}^{(k 0)}}{G_{I I c}}-1 \geq 0
$$

$G_{I}^{(k 0)}, G_{I I}^{(k 0)}$ are energy release rates due to modes $I, I I$ crack propagation in the ply $(k 0)$ of the laminate. Even if $G_{I, I I}^{(k 0)}$ are calculated at global level of the laminate, considering the contribution of all $(k)$ plies to the energy released due to crack growth in the current $(k 0)$ cracking ply, subscripts $(k 0)$ are used in eq. (11) in order to emphasize that energy release rates are evaluated due to damage multiplication in the current cracking ply $(k 0)$ only. $G_{I c}, G_{I I c}$ are critical values of the energy release rates, or the material resistance to modes $I$ and $I I$ crack propagation; $G_{I c}$ and $G_{I c}$ are regarded as material properties whose evaluation is based on experiments.

For displacement control conditions, the strain ERR, $G$, is

$$
G=-\frac{\Delta U}{\Delta A}
$$

where $\Delta U$ is the strain energy released by the new crack formation, and $\Delta A$ is the new formed crack area. The strain energy released due to incremental crack multiplication is calculated as the difference between the two instances of the material, before and after crack growth: $\Delta U=U^{\text {after }}-U^{\text {before }}$.

For the calculation of the strain energy before/after crack multiplication, and the partition of the total strain energy in modes I/II crack growth [27] as required by the crack growth criterion in eq. (11), the strain energy density stored in the ply $(k)$ of the laminate is written as

$$
U_{0}^{(k)}=\frac{1}{2}\left\{\varepsilon^{(k)}\right\}^{T} \cdot\left[\bar{Q}^{(k)}\right] \cdot\left\{\varepsilon^{(k)}\right\}
$$

where $\varepsilon^{(k)}$ is the vector of the strain components of the ply $(k)$, written in global (laminate) c.s. However, the $G_{I / I I}^{(k 0)}$ in eq. (11) represent the I/II modes components of ERR of the laminate due to crack multiplication in the ply $(k 0)$, where strain energy contribution from all plies $(k)$ to the crack multiplication in ply $(k 0)$ is taken into account. For this, the $\varepsilon^{(k)}$ strain of ply $(k)$ is first transformed to the c.s. of the cracking ply $(k 0)$, and then separated in components corresponding to the ERR for modes $I$ and II crack multiplication in ply $(k 0)$

$$
\begin{aligned}
\varepsilon^{(k)} & =\left\{\varepsilon_{11}, \varepsilon_{22}, \varepsilon_{12}\right\}^{(k)} \\
& \Rightarrow\left\{\begin{array}{l}
\varepsilon_{I}^{(k)}=\left\{0, \varepsilon_{22}, 0\right\}^{(k)} \\
\varepsilon_{I I}^{(k)}=\left\{0,0, \varepsilon_{12}\right\}^{(k)}
\end{array}\right.
\end{aligned}
$$

These mode components are then used for the calculation of strain energy density in eq. (13), and the strain energy of the whole laminate for crack formation in the cracking ply $(k O)$ is then expressed as the summation of the available strain energy in each ply $(k), U_{I / I I}^{(k 0)}=\sum_{k=1}^{N} U_{I / I I}^{(k)}$.

The effect of the thermal residual stresses in the laminate, which are the result of cool-down after curing and the thermal expansion coefficient mismatch between different plies of the laminate, is included in the model by considering in eq. (13) the strain quantity that effectively gives strain energy of deformation in ply $(k)$

$$
\varepsilon^{(k)}=\varepsilon_{t o t}^{(k)}-\alpha^{(k)} \cdot \Delta T
$$

where $\varepsilon_{\text {tot }}^{(k)}$ represents the total deformation of ply $(k)$, due to both mechanical and thermal loading.

The bending deformation is included by considering the linear variation of strain over the laminate thickness 


$$
\varepsilon_{\text {tot }}^{(k)}=\varepsilon_{\text {tot }}^{(0)}+z \cdot k_{t o t}
$$

The crack closure effect, that has to be accounted for in flexural deformation, is considered according to the sign of the transverse stress in ply $(k)$

$$
\rho_{i+1}^{(k)}= \begin{cases}(i+2) \rho_{0} & \text { if } \sigma_{22}^{(k)}>0 ; \text { crack is open (active) } \\ 0 & \text { if } \sigma_{22}^{(k)} \leq 0 ; \text { crack is close (pasive) }\end{cases}
$$

The experimentally observed $R$-curve behavior of the material resistance to crack multiplication [28] is also implemented in the present model. This phenomenon, that is specific to laminated composites, means an increasing resistance $G_{c}$ of the material to crack growth with increasing damage level; it is attributed to the fiber bridging effect [29] during matrix crack multiplication process. According to this effect, the critical ERR in eq. (11) becomes $G_{c}=G_{c}(\lambda)$, where $\lambda$ is the current damage level (crack density) of the ply. Moreover, for laminated composites there is also a thickness dependence of $G_{c}$ [28], which is denoted in this model as the in-situ R-curve behavior. According to this, the critical ERR becomes $G_{c}=G_{c}(\lambda, t)$ [24], [25]. In this work, only mode $I$ critical SEER is accounted for the in-situ R-curve effect; for mode $I I$, a constant value of $G_{c}$ is used, due to lack of experimental data.

A first set of matrix cracking onset and multiplication experimental data, for a reference laminate having a cracking ply of thickness $t=t_{\text {ref }}$, is used to adjust the $R$-curve behavior of the reference laminate, which is modeled by the equation

$$
G_{I c}\left(\lambda, t=t_{r e f}\right)=G_{I c}^{r e f}=G_{I c, 0}^{r e f}+\tan \left(\beta_{\lambda}^{r e f}\right) \cdot \lambda
$$

where $G_{I c, 0}^{r e f}$ critical ERR for onset of damage (the advent of the first matrix cracking), and $\beta_{\lambda}^{\text {ref }}$ is a parameter that describes the rate of damage growth, with $0 \leq \beta_{\lambda}^{\text {ref }}<90$. Both $G_{I c, 0}^{r e f}$ and $\beta_{\lambda}^{\text {ref }}$ parameters are identified by reduction of experimental data of the reference laminate.

A second set of matrix cracking onset and multiplication experimental data, for a laminate having a cracking ply of thickness $t \neq t_{\text {ref }}$, is considered next, in order to capture the in-situ (thickness) effect of the fracture toughness. Based on this, the onset fracture toughness is modeled as

$$
G_{I c, 0}(t)=G_{I c, 0}^{r e f} \cdot\left\lfloor 1+\left(\frac{t}{t_{r e f}}-1\right)\left(\beta_{0}^{t}-1\right)\right\rfloor
$$

where $\beta_{0}^{t}$ is an in-situ onset reduction data parameter, with $1 \leq \beta_{0}^{t} \leq 2$. Finally, the in-situ $R$-curve of the given material can be modeled based on the equation

$$
\begin{aligned}
G_{I c}(\lambda, t) & =G_{I c, 0}(t)+ \\
+ & {\left[1+\left(\frac{t}{t_{r e f}}-1\right)\left(\beta_{\lambda}^{t}-1\right)\right] \cdot \tan \left(\beta_{\lambda}^{r e f}\right) \cdot \lambda }
\end{aligned}
$$

where $\beta_{\lambda}^{t}$ is an in-situ growth reduction data parameter, with $1 \leq \beta_{\lambda}^{t} \leq 2$.

The output of the progressive matrix cracking analytical model is:

- the reduced material properties of the laminate as a function of damage level in any ply $(k): C=C\left(\lambda^{(k)}\right)$;

- the curve of damage multiplication under external loading for each ply (k) of the laminate: $\lambda^{(k)}=\lambda^{(k)}(\varepsilon)$;

- the curve of reduction in thermo-elastic properties of the laminated composite under external loading: $C=C(\varepsilon)$;

- the nonlinear constitutive stress--strain behavior of the laminate material: $\sigma=\sigma(\varepsilon)$.

\section{RESUlTS AND CONCLUSIONS}

The progressive damage matrix cracking experimental results presented in [18] are used here for the validation of the analytical model predictions. The material is a Glass/Epoxy Fibredux 913G-E-5-30\% system, having the following thermo-elastic properties of individual plies: $E_{1}=34 \mathrm{GPa}, E_{2}$ $=18 \mathrm{GPa}, G_{12}=7.9 \mathrm{GPa}, v_{12}=0.29, v_{23}=0.41, \alpha_{1}=$ $6.72 \times 10^{-6}{ }^{\circ} \mathrm{C}, \alpha_{2}=29.3 \times 10^{-6}{ }^{\circ} \mathrm{C}$. The ply thickness is $t_{k}=$ $0.125 \mathrm{~mm}$, and the processing temperature (stress free temperature) is $S F T=125^{\circ} \mathrm{C}$. Two experimental data sets, namely $[0 / 90 / 0]$ and $\left[0 / 90_{2} / 0\right]$ are used for the calculation of the in-situ $R$-curve parameters, yielding the following values: $G_{I c, 0}^{r e f}=0.2 \mathrm{~N} / \mathrm{mm}, \beta_{\lambda}^{r e f}=0 \mathrm{deg}, \beta_{0}^{t}=1, \beta_{\lambda}^{t}=1$.

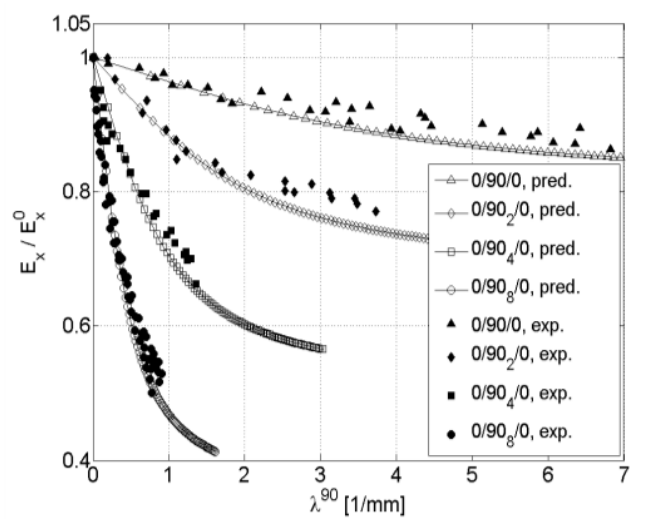

Fig. 1. Variation of normalized Young modulus with crack density; experimental data from [18].

A first set of experimental results is presented in [18] for the $\left[0 / 90_{\mathrm{n}} / 0\right]$ LSS under uniaxial extension $\varepsilon_{x}$. Reduced material properties of the laminate are presented in Fig. 1, 2, for the Young modulus and Poisson ratio, respectively. Good agreement between analytical and experimental results can be noticed in the two plots. However, it can also be noticed the model tendency of predicting a lower slope of the 
analytical trend compared to the experimental trend, at high values of the crack density $\lambda$, especially for high thickness of the cracking plies. The matrix cracking evolution curves are presented in Fig. 3. Again, the least favorable match of the experimental data can be noticed for the thick cracking plies, at high crack densities.

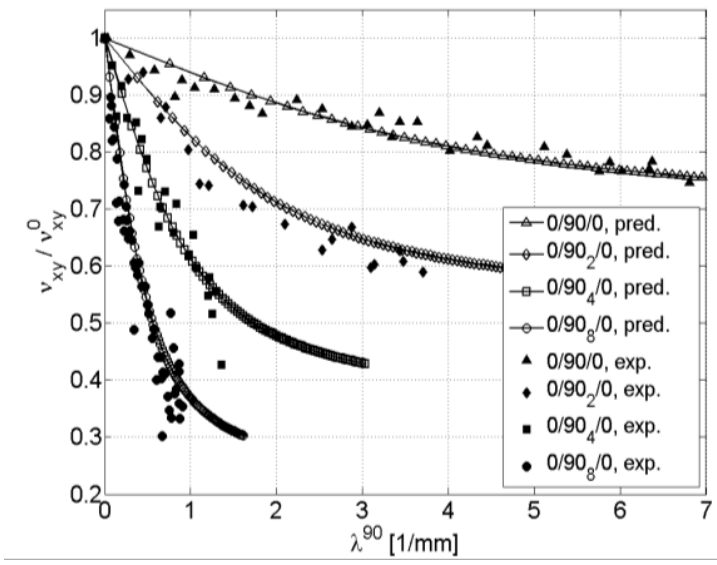

Fig. 2. Variation of normalized Poisson ratio with crack density; experimental data from [18].

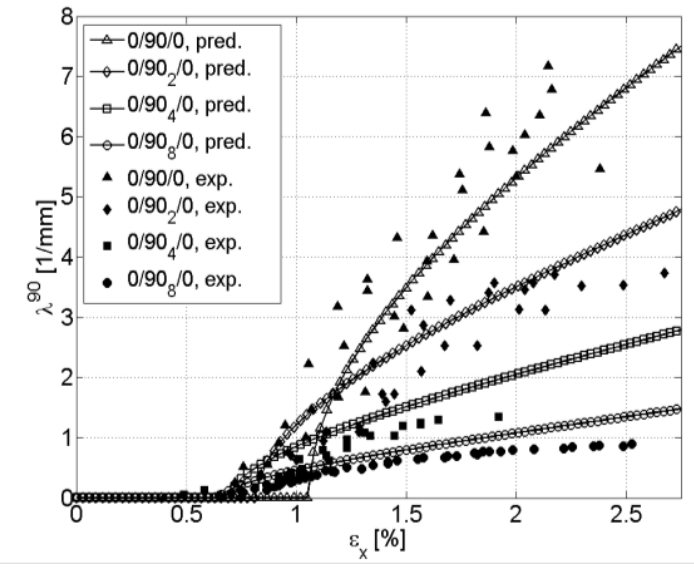

Fig. 3. Variation of crack density with applied strain; experimental data from [18].

Stress-strain curves featuring nonlinearities due to matrix cracking are shown in Fig. 4, where good agreement between analytical and experimental results is obtained. The least favorable correlation is obtained for the $\left[0 / 90_{8} / 0\right]$ laminate featuring the thickest cracking ply, which is in connection with the least favorable correlation of damage evolution curve in Fig.3. This deteriorating predicting capability of the model at high thickness of the cracking ply could be due to the so called transition thickness effect, experimentally noticed for laminated composites: there is a certain thickness (i.e., the transition thickness) of the ply, over which the strain at matrix cracking onset remains constant.

Experimental data for matrix cracking under flexural loading is very rare in the literature. A set of such data is presented in [18], for the LSS $\left[90_{2} / 0_{2} /-45_{2} / 45_{2}\right]_{S}$ and $\left[90_{2} /-45_{2} / 45_{2}\right]_{S}$. The curves of distributed bending moment-curvature (which are the equivalent of the stress-strain plots for the case of extension loading) are presented in Fig. 5, where good agreement between analytical predictions and experiments can be noticed.

Model prediction for combined extension-bending loading $\varepsilon_{x}-k_{x}$ is presented in Fig. 6. The LSS considered for this case is $\left[0 / 90_{2} / 0 / 90_{3} / 0\right]_{S}$. Without using the symmetry notation, the laminate is denoted as $\left[0 / 90_{2}^{(1)} / 0 / 90_{3}^{(1)} / 0_{2} / 90_{3}^{(2)} / 0 / 90_{2}^{(2)} / 0\right]$; the superscripts (1) denotes plies at the bottom of the LSS (which would be under compression if only bending deformation was applied), and the superscripts (2) denotes plies at the top of the LSS (which would be under tension if only bending deformation was applied). The applied loading is simultaneous extension $\varepsilon_{x}$ and bending $k_{x}$, under displacement control; the ratio of the two applied incremental deformations is $k_{x}=0.5 \cdot \varepsilon_{x}$ for the present study.

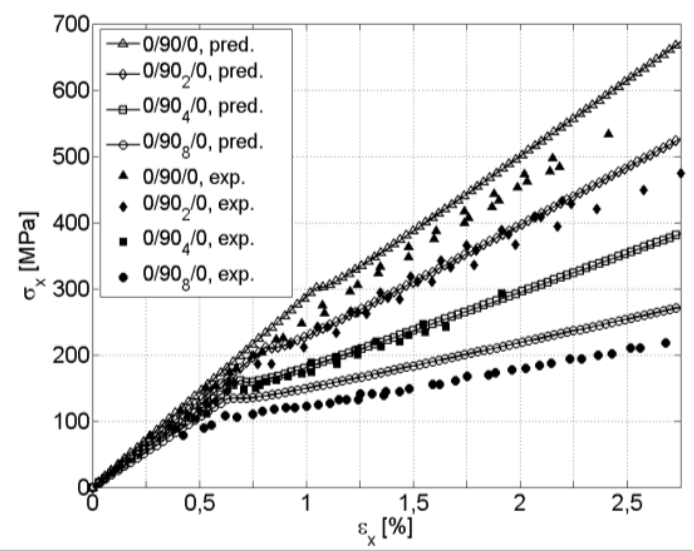

Fig. 4. Non-linear stress-strain curves due to matrix cracking; experimental data from [18].

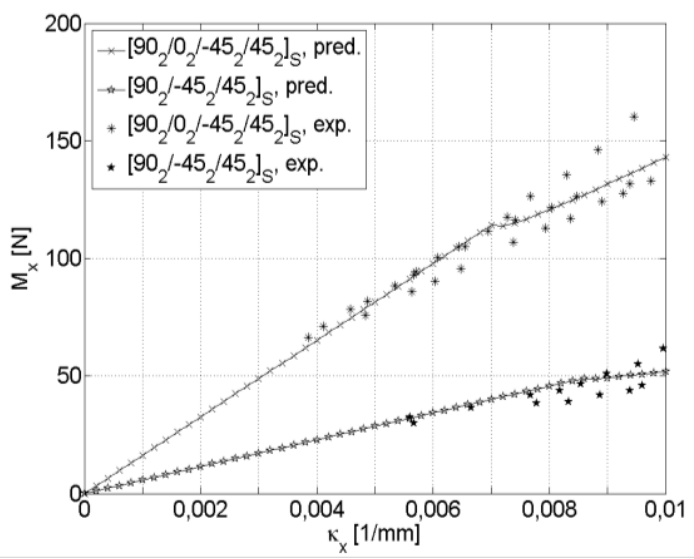

Fig. 5. Non-linear distributed bending moment vs. curvature due to matrix cracking; experimental data from [18].

It can be observed in Fig. 6 that the cracking process initiates almost simultaneous in the top $90_{2}^{(2)}$ and $90_{3}^{(2)}$ plies, due to combined effect of ply thickness and bending deformation. Regarding the damage process in the bottom $90_{2}^{(1)}$ and $90_{3}^{(1)}$ plies, it can be observed that there is matrix cracking in the $90_{3}^{(1)}$ ply, and there is no matrix cracking in the $90_{2}^{(1)}$ ply. The extensional strain exceeds the negative bending strain due to bending in both plies. However, there is more net strain in the $90_{3}^{(1)}$ ply than in the $90_{2}^{(1)}$ ply because of their position in the LSS, and the $90_{3}^{(1)}$ is weaker because it is thicker (in-situ effect). This is the reason why matrix 
cracking appears in the $90_{3}^{(1)}$ ply and it does not appear in the $90_{2}^{(1)}$ ply.

In conclusion, the present model for progressive matrix cracking of general laminates, under combined membrane-flexural deformation, accounts for multiple aspects of the problem: the effect of thermal residual stresses, the crack closure effect in bending, and the in-situ R-curve effect. Two experimental data sets of damage growth, for two laminate configurations of the same material system, are needed in order to fit the R-curve parameters; then, the model is able to predict damage onset and evolution for other LSS. There is no limitation on the configuration of the laminate or on the number of the cracking plies, as it is the case of the most models available in the literature, where only symmetric LSS are addressed, or only certain plies can undergo matrix cracking. The trends output by the analytical model, as well the comparison against experimental data, provides confidence in the predicting capabilities on the proposed model.

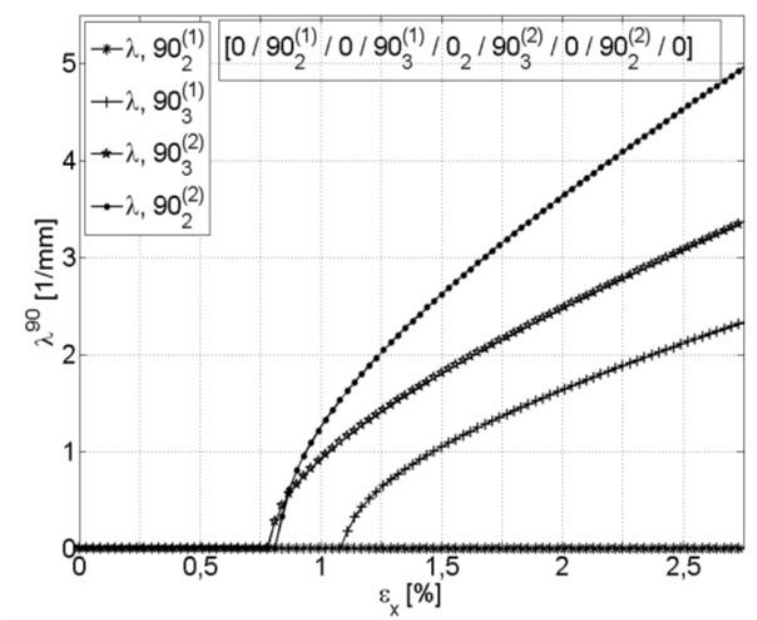

Fig. 6 . Analytical variation of crack density for combined membrane-flexural loading.

\section{ACKNOWLEDGMENT}

The financial support by the Austrian Federal Ministry of Science, Research and Economy and the National Foundation for Research, Technology and Development is gratefully acknowledged.

\section{REFERENCES}

[1] J. A. Nairn and D. A. Mendels, "On the use of planar shear-lag methods for stress-transfer analysis of multilayered composites," Mechanics of Materials, vol. 33, no. 6, pp. 335-362, 2001.

[2] J. A. Nairn, "Finite fracture mechanics of matrix microcracking in composites," Application of Fracture Mechanics to Polymers, Adhesives and Composites, Elsevier, pp. 207-212, 2004.

[3] J. A. Mayugo, P. P. Camanho, P. Maimi, and C. G. Da'vila, "Analytical modeling of transverse matrix cracking of $[ \pm \theta / 90 \mathrm{n}]$ composite laminates under multiaxial loading," Mechanics of Advanced Materials and Structures, vol. 17, pp. 237-245, 2010.

[4] R. J. Nuismer and S. C. Tan, "Constitutive relations of a cracked composite lamina," Journal of Composite Materials, vol. 22, no. 4, pp. 306-321, April 1988.

[5] S. C. Tan and R. J. Nuismer, "A theory for progressive matrix cracking in composite laminates," Journal of Composite Materials, vol. 23, no. 10, pp. 1029-1047, October 1989.

[6] S. Li, S. R. Reid, and P. D. Soden, "A continuum damage model for transverse matrix cracking in laminated fibre-reinforced composites," Mathematical, Physical and Engineering Sciences, vol. 356, no. 1746 , pp. 2379- 2412, October 1998.
[7] T. Yokozeki and T. Aoki, "Stress analysis of symmetric laminates with obliquely-crossed matrix cracks," Advanced Composite Materials, vol. 13, no. 2, pp. 121-140, 2004.

[8] T. Yokozeki and T. Aoki, "Overall thermoelastic properties of symmetric laminates containing obliquely crossed matrix cracks," Composites Science and Technology, vol. 65, no. 11-12, pp. 1647-1654, 2005.

[9] D. H. Cortes and E. J. Barbero, "Stiffness reduction and fracture evolution of oblique matrix cracks in composite laminates," Annals of Solids and Structural Mechanics, vol. 1, no. 1, pp. 29-40, 2010.

[10] J. Varna, R. Joffe, N. V. Akshantala, and R. Talreja, "Damage in composite laminates with off-axis plies," Composites Science and Technology, vol. 59, no. 14, pp. 2139-2147, 1999.

[11] J. Varna, R. Joffe, and R. Talreja, "A synergistic damage-mechanics analysis of transverse cracking in $[ \pm \theta / 904]$ s laminates," Composites Science and Technology, vol. 61, no. 5, pp. 657-665, 2001.

[12] E. J. Barbero and D. H. Cortes, "A mechanistic model for transverse damage initiation, evolution, and stiffness reduction in laminated composites," Composites Part B, vol. 41, pp. 124-132, 2010.

[13] E. J. Barbero, G. Sgambitterra, A. Adumitroaie, and X. Martinez, "A discrete constitutive model for transverse and shear damage of symmetric laminates with arbitrary stacking sequence," Composite Structures, vol. 93, no. 2, pp. 1021-1030, January 2011.

[14] E. Adolfsson and P. Gudmundson, "Thermoelastic properties in combined bending and extension of thin composite laminates with transverse matrix cracks," International Journal of Solids and Structures, vol. 34, no. 16, pp. 2035-2060, June 1997.

[15] T. Yokozeki, T. Aoki, and T. Ishikawa, "Consecutive matrix cracking in contiguous plies of composite laminates," International Journal of Solids and Structures, vol. 42, no.9-10, pp. 2785-2802, May 2005.

[16] T. Yokozeki, T. Aoki, T. Ogasawara, and T. Ishikawa, "Effects of layup angle and ply thickness on matrix crack interaction in contiguous plies of composite laminates," Composites Part A (Applied Science and Manufacturing), vol. 36, no. 9, pp. 1229-1235, 2005.

[17] G. Sgambitterra, A. Adumitroaie, E. J. Barbero, and A. Tessler, "A robust three-node shell element for laminated composites with matrix damage," Composites Part B: Engineering, vol. 42, no.1, pp. 41-50, 2011.

[18] E. Adolfsson and P. Gudmundson, "Matrix crack initiation and progression in composite laminates subjected to bending and extension," International Journal of Solids and Structures, vol. 36, no. 21, pp. 3131-3169, 1999

[19] E. J. Barbero, Introduction to Composite Materials Design, 2nd ed. Philadelphia, PA, USA: CRC Press, 2011.

[20] E. J. Barbero, Workbook for Introduction to Composite Materials Design, South Charleston, SC, USA: CreateSpace, 2015.

[21] P. Gudmundson and S. Ostlund, "Prediction of thermoelastic properties of composite laminates with matrix cracks," Composites Science and Technology, vol. 44, no. 2, pp. 95-105, 1992.

[22] P. Gudmundson and W. Zang, "An analytic model for thermoelastic properties of composite laminates containing transverse matrix cracks," International Journal of Solids and Structures, vol. 30, no. 23 , pp. 3211-3231, 1993.

[23] W. Zang and P. Gudmundson, "Damage evolution and thermoelastic properties of composite laminates," International Journal of Damage Mechanics, vol. 2, no. 3, pp. 290-308, July 1993.

[24] A. Adumitroaie, "Initiation and evolution of matrix cracking in nonsymmetric laminates under in-plane and flexural loading," PhD Thesis, Dep. Mech. Aerosp. Eng., West Virginia University, Morgantown, WV, 2012.

[25] A. Adumitroaie and E. J. Barbero. (2015). Intralaminar damage mode for laminates subjected to membrane and flexural deformations. Mechanics of Advanced Composite Materials and Structures. [Online]. Available: http://dx.doi.org/10.1080/15376494.2013.796541

[26] H. T. Hahn, "A mixed-mode fracture criterion for composite materials," Composites Technology Review, vol. 5, pp. 26-29, 1983.

[27] J. Rebiere and D. Gamby, "A decomposition of the strain energy release rate associated with the initiation of transverse cracking, longitudinal cracking and delamination in cross-ply laminates,' Composite Structures, vol. 84, no. 2, pp. 186-197, 2008.

[28] D. T. G. Katerelos, J. Varna, and C. Galiotis, "Energy criterion for modeling damage evolution in cross-ply composite laminates," Composites Science and Technology, vol. 68, pp. 2318-2324, 2008.

[29] B. F. Sorensen and T. K. Jacobsen, "Large-scale bridging in composites: R-curves and bridging laws," Composites Part A (Applied Science and Manufacturing), vol. 29A, no. 11, pp. 1443-1451, 1998. 


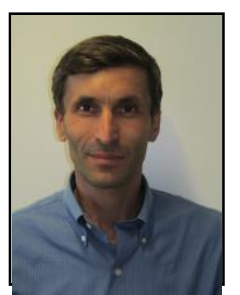

Adi Adumitroaie was born in Romania. $\mathrm{He}$ is currently a research associate at the Institute for Constructional Lightweight Design, Johannes Kepler University, Linz, Austria. He has earned the degree of $\mathrm{Ph} . \mathrm{D}$. in aerospace engineering (2013) from West Virginia University, Morgantown, West Virginia, USA. His education also includes the degree of MSc. Eng. in mechanical engineering (2003) with Politehnica University of Bucharest, Romania, and the degree of B.Eng. in aerospace engineering with the Military Technical Academy, Bucharest, Romania. His previous work experience includes the positions of FEA engineer with Ansys Inc., Canonsburg, Pennsylvania, USA, and aeronautical engineer with Romanian Air Forces, Romania.

His main research interest is in the field of composite materials for lightweight structures, including aspects of material constitutive behavior and stress analysis, nonlinear structural behavior and stability, fracture mechanics, inter-laminar and intra-laminar progressive damage, structural damage tolerance under static, dynamic, and fatigue loading. Both aspects of analytical and numerical (FEA) modeling are of interest.

Dr. Adumitroaie has published with known international journals based on his research work in fabric reinforced flexible composite with application to inflatable structures, and progressive damage in structural long fiber reinforced polymers.

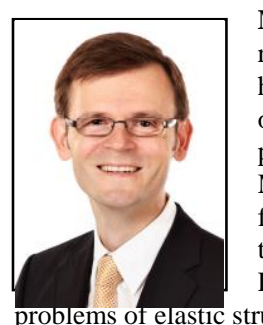

Martin Schagerl was born in Vienna, Austria. He received his Ph.D. in mechanical engineering and a habilitation in mechanics from the Vienna University of Technology. From 1995 to 2002 he held the position of university assistant at the Institute of Mechanics at this university. Awarded a Max-Kade fellowship he visited the Mathematics Department of the University of Maryland, USA, in 2000/2001. Publications from this period treat mainly non-linear problems of elastic structures by geometrically exact theories.
In 2002 he started to work in the structural analysis department of the aircraft manufacturer Airbus in Hamburg, Germany. In 2009, he was appointed full professor and head of the Institute of Constructional Lightweight Design at Johannes Kepler University Linz, Austria. Since 2014 he is also head of the Christian Doppler Laboratory for Structural Strength Control of Lightweight Constructions. In these positions his current research activities focus on static strength and fatigue life analysis of lightweight structures, effects of damages and manufacturing defects in composite materials, and mechatronic systems for structural health monitoring.

Prof. Schagerl is a member and since 2006 chair of the Industry Committee for Structural Analysis, the expert and editorial board of the Handbook Structure Analysis HSB.

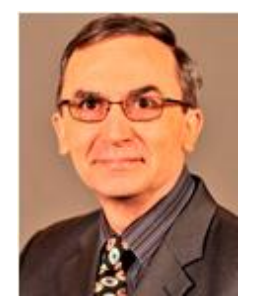

Ever J. Barbero received a BSME/BSEE degree from UNRC (Argentina) and a Ph.D. in engineering mechanics from Virginia Tech (USA). He is a fellow of ASME and SAMPE, professor of mechanical and aerospace engineering at West Virginia University, and honorary professor at Universidad Carlos III de Madrid (Spain), University of Puerto Rico (PR), and Universidad Nacional de Trujillo (Peru).

His research interests are broad, including constitutive modeling, material characterization, structural design, multifunctional/multiscale/multyphysics simulation, inflatable structures, and multiphysics composite materials. He is the author of several books and book chapters, many journal publications, numerous conference papers, and mentor of over $30 \mathrm{MS}$ and $15 \mathrm{Ph} . \mathrm{D}$. graduates currently serving leadership positions in academia and industry worldwide. He holds two US Patents, $\# 6,455,131$ (2002) and \#6,544,624 (2003), received the Aerospace Alumn Academy Award for Outstanding Teaching, and numerous research awards. $\mathrm{He}$ is Associate Editor for Annals of Solid and Structural Mechanics (Springer) and Member of the Editorial Board of Revista Internacional de Desastres Naturales, Accidentes e Infraestructura Civil (University of Puerto Rico). 